\title{
ON THE EXTENSIONS OF OPTIMAL CONTROL THEORY
}

\author{
Dmitry Karamzin*, Valeriano de Oliveira ${ }^{\dagger}$, Fernando Pereira $^{\ddagger}$, Geraldo Silva ${ }^{\S}$ \\ * Universidade Estadual Paulista (IBILCE UNESP), Sao Jose do Rio Preto, SP, Brazil \\ ${ }^{\dagger}$ Universidade Estadual Paulista (IBILCE UNESP), Sao Jose do Rio Preto, SP, Brazil \\ $\ddagger$ University of Porto, FEUP, Porto, Portugal \\ $\S$ Universidade Estadual Paulista (IBILCE UNESP), Sao Jose do Rio Preto, SP, Brazil \\ Emails: dmitry_karamzin@mail.ru, antunes@ibilce.unesp.br, flp@fe.up.pt, \\ gnsilva@ibilce.unesp.br
}

\begin{abstract}
We consider an impulsive control problem with state constraints which arises as result of extension of the classic theory. Filippov's type existence theorem is proved.
\end{abstract}

Keywords - impulsive control, existence theorems, state constraints.

\section{Introduction}

It is a fact that classical calculus of variations problems might not have a smooth or even a continuous solution, although they are still of physical interest. Here, we shall focus namely on the discontinuous case. The following problem of variational calculus illustrates how discontinuities may emerge.

$$
\begin{array}{cl}
\text { Minimize } & \int_{0}^{1} x(t) \sqrt{1+(\dot{x})^{2}} d t, \\
\text { subject to } & x(0)=R_{1}, x(1)=R_{2} .
\end{array}
$$

This is the problem of finding a minimal area of the surface of revolution formed by a membrane stretched over two parallel disks of radiuses $R_{1}$ and $R_{2}$, respectively. The application of the Euler-Lagrange principle leads to a second order differential equation and a boundary problem, which does not have a solution for some values of the parameters $R_{1}$, and $R_{2}$. The physical meaning of such situation is as follows: if numbers $R_{1}$, and $R_{2}$ are sufficiently large (or the distance between the two disks sufficiently small), the membrane exists and the surface of revolution is smooth. But, as the distance between the two disks increases, the membrane stretches and, at some point, breaks: at that very moment, the smooth and continuous solution fails to exist. However, this does not mean that the surface of revolution does not exist at all. Clearly, it is the surface of the union of the two disks aimed at each other and the segment $[0,1]$. This means that the solution $x(t)$ will be $R_{1}$ for $t=0, R_{2}$ for $t=1$ and 0 for $t \in(0,1)$ and, thus, it is discontinuous. In other words, the solution will be impulsive.

In the framework of his famous program David Hilbert suggested to extend calculus of variations theory in order to cover and to formulate such degenerate situations by giving a strict mathematical meaning to non-classical solutions. He expressed confidence that "every problem in the calculus of variations has a solution, provided that the term 'solution' is interpreted appropriately". This desideratum spawned a number of developments on the extension of the classic calculus of variation by various authors. For the rich history of this issue, we refer the reader to the article (B. Mordukhovich, Existence of optimal controls. J. Soviet Math. 7 (1977), 850-886). Here, we only point out to important contributions on extensions of the classical calculus of variations made by Lebesgue, Tonelli, Young, Bogolyubov, Gamkrelidze, Krotov, Rockafellar, Tikhomirov, Warga, among others.

With the advent of Optimal Control and the Pontryagin's maximum principle in the fifties, the theory of discontinuous solutions for variational calculus advanced significantly gradually giving shape to the area of Impulsive Optimal Control. So, what is the subject of the impulsive control theory? This theory covers and contains in itself, as a limiting case, a wide class of degenerate calculus of variations and optimal control problems for which classical continuous solutions fail to exist. This theory provides not only the way how to interpret the concept of solution but also the procedure to find it. The basic idea is to extend the conventional concept of control as well as the concept of trajectory. The usual bounded and measurable control can be replaced, for instance, by a Borel measure. Then, the trajectory becomes a function of bounded variation. This approach already suggests a reasonable extension for linear systems which covers many actual applications.

Let us give a simple example to illustrate how impulsive controls arise by providing an extension to the following calculus variation problem

$$
\begin{array}{cl}
\text { Minimize } & \int_{0}^{1} x^{2} d t, \\
\text { subject to } & \dot{x}=v, v \in \mathbb{R}^{1}, \\
& x(0)=0, x(1)=1 .
\end{array}
$$


So, we need to minimize the area under the curve $x^{2}(\cdot)$ where the arc $x(\cdot)$ is to reach the point $x=1$ starting from zero at $t=0$. Then, there is obviously no solution to this problem in the class of continuous trajectories due to the fact that any minimizing sequence of trajectories converges pointwise to the discontinuous function $x(t)=0$ as $t \in[0,1)$ and $x(1)=1$.

Then, how could a solution be defined? A solution can be found by extending the set of admissible trajectories admitting that the trajectory might have now discontinuities. Or, equivalently, this means to introduce impulsive controls instead of the convenient integrable control $v$ from $\mathbb{L}_{1}$. For problem (2), it is appropriate to consider Borel measures as impulsive controls. Any conventional control $v(\cdot)$ can be considered as an absolutely continuous measure $\mu$ such that $d \mu=v(t) d t$. However, there will exist also other controls, like Dirac's measures, that cannot be reduced to conventional ones.

Thus, the conventional problem is extended by enlarging the class of trajectories/controls, being problem (2) rewritten in the impulsive context as follows:

$$
\begin{array}{cl}
\text { Minimize } & \int_{[0,1]} x^{2} d t, \\
\text { subject to } & d x=d \mu, \quad \mu \in C^{*}([0,1]), \\
& x(0)=0, x(1)=1 .
\end{array}
$$

Here, any admissible trajectory $x(\cdot)$ is already a function of bounded variation and so may exhibit discontinuities. The notation $d x=d \mu$ is understood in the integral sense, or, in terms of measures, it means that the Borel measure generated by $x(\cdot)$ is absolutely continuous with respect to $\mu$ and it is its Radon-Nykodim derivative with respect to $\mu$ (which, in this particular case, is equal to unity).

It is easy to see that solution to the extended problem exists and the optimal trajectory is $x(t)=0$ for $t<1$, and $x(1)=1$. Moreover, the fact that Borel measures are being used as extended controls, together with the weakly* sequential compactness of the unit ball in $C^{*}([0,1])$, implies that the described extension procedure is successful in ensuring the existence of solution for large classes of linear control problems. (For example, whenever the total variation $\int_{0}^{1}|v(t)| d t$ is to be minimized.)

Thanks to the weak* convergence of measures, the extension procedure is rather clear when the dynamical system is linear in $(x, v)$, like in the example (2). However, the complexity of the extension will increase when more general dynamical control systems are considered:

$$
\dot{x}=f(x, u, t)+g(x, t) v, \quad v \in K,
$$

where $u$ is usual (classical) bounded control, the function $f$ defines conventional control dynamics, $v$ is an unbounded vector-valued control, $g$ some matrix-valued function, and the set $K$, a convex closed cone.

How to describe the solution in this case? The extension procedure introduced above can not be applied any longer since the passage to the weak* limit is not correct for non-linear systems. Indeed, this is shown by the following simple example. Let $K=\mathbb{R}^{2}$. Consider the dynamical system with vector-valued control $v=\left(v_{1}, v_{2}\right)$ :

$$
\dot{x}=x v_{1}+x^{2} v_{2}, \quad x(0)=1 .
$$

If we try to extend this system to the class of Borel measures, regarding bounded total variation $\int_{0}^{1}|v(t)| d t \leq$ const, we will see that, to every control, i.e., to every vector measure, there corresponds an entire integral funnel of trajectories $x(\cdot)$, any of which may claim to be called a solution to the extended dynamical system.

Thus, in the non-linear case, Borel measures are simply not enough to construct all achievable trajectories and controls. But, as we will see later, the new design control turns out be a Borel measure plus a certain family of usual measurable functions, which we will designate by associated family. The reason to introduce these associated functions is to select a single trajectory from the integral funnel and, thus, they can be regarded as controls acting at the discontinuities of the trajectory.

Therefore, herein, we aim at extending the classic calculus of variations and/or optimal control problems by introducing a new type of impulsive controls. We provide appropriate theorems for the existence of solution for constrained impulsive control problems.

Before concluding the introduction, let us show that there is no loss of generality in considering the case in which the cone $K$ in (3) is contained in the first orthant $\mathbb{R}_{+}^{k}:=\left\{\xi \in \mathbb{R}^{k}: \xi^{j} \geq 0\right\}$. Indeed, along with the system (3) we consider the system

$$
\dot{x}=f(x, u, t)+g(x, t) P \bar{v}, \quad \bar{v} \in \bar{K} .
$$

Here, $P: \mathbb{R}^{2 k} \rightarrow \mathbb{R}^{k}$ is a linear operator defined by $(P \xi)^{j}=\xi^{2 j}-\xi^{2 j-1}, j=1, \ldots, k$, and $\bar{K}=\{\bar{v} \in$ $\left.\mathbb{R}_{+}^{2 k}: P \bar{v} \in K\right\}$ is a closed convex cone.

Note that any scalar function $r(t)$ can be represented as a difference of two non-negative functions, i.e., $r=r^{+}-r^{-}$, where $r^{+}(t)=$ $\max \{r(t), 0\}$, and $r^{-}(t)=-\min \{r(t), 0\}$. Then, for a given function $\bar{v}$, we define $v$ as $v=P \bar{v}$, and, vice-versa, for a given function $v$, we construct $\bar{v}$ by the formula $\bar{v}^{2 j-1}=v^{j-}, \bar{v}^{2 j}=v^{j+}$, $j=1, \ldots, k$. This establishes the equivalence of the systems (3) and (4) in the sense that the sets of admissible trajectories for (3), and (4) coincide. 
At the same time, this equivalence is ambiguous since one control $v$ may correspond to different controls $\bar{v}$. Nevertheless, all such $\bar{v}$ will generate the same trajectory.

\section{Statement of the Problem}

Consider the following optimal impulsive control problem:

$$
\begin{cases}\text { Minimize } & c(p) \\ \text { subject to } & d x=f(x, u, t) d t+g(x, t) d \vartheta \\ & d y=d|\vartheta|, y\left(t_{0}\right)=0 \\ & \varphi(x, t) \leq 0 \\ & u(t) \in U, \operatorname{range}(\vartheta) \subset K \\ & p \in S, t \in T .\end{cases}
$$

Here, the functions $c: \mathbb{R}^{2 n+1} \rightarrow \mathbb{R}^{1}, f: \mathbb{R}^{n} \times \mathbb{R}^{m} \times$ $\mathbb{R}^{1} \rightarrow \mathbb{R}^{n}, g: \mathbb{R}^{n} \times \mathbb{R}^{1} \rightarrow \mathbb{R}^{n \times k}, \varphi: \mathbb{R}^{n} \times \mathbb{R}^{1} \rightarrow \mathbb{R}^{l}$ are continuously differentiable, $T=\left[t_{0}, t_{1}\right]$ is a fixed time interval, $p=\left(x_{0}, x_{1}, y_{1}\right)$, where $x_{0}=$ $x\left(t_{0}\right), x_{1}=x\left(t_{1}\right)$ and $y_{1}=y\left(t_{1}\right)$, is the so called endpoint vector, $S$ is a closed set in $\mathbb{R}^{2 n+1}, U$ is a closed set in $\mathbb{R}^{m}, K$ is a closed convex cone contained in the first orthant $\mathbb{R}_{+}^{k}, c(p)$ is the cost function to be minimized, the vector-function $\varphi$ defines the inequality state constraints, and $\vartheta=\left(\mu ;\left\{v_{\tau}\right\}\right)$ is a new object which we call impulsive control. The measure $|\vartheta|$ designates the variation measure of the impulsive control, whereas $y_{1}$ becomes its total variation.

The impulsive control consists of two components. While the first one, $\mu$, is a vector Borel measure with range in $K$ (this means that $\mu(B) \in$ $K$ for any Borel set $B \subset T)$, the second component, $\left\{v_{\tau}\right\}$, is a family of measurable vector functions defined on the interval $[0,1]$, with values in $K$, and depending on the real parameter $\tau \in T$. Let us provide the exact properties of this family as well as the definition of the impulsive control and of the associated trajectory $x(\cdot)$, that solves to the differential equation in (5).

Consider a Borel vector measure $\mu$ such that range $(\mu) \subset K$. Take a number $\tau \in T$. Let $W_{\tau}(\mu) \subset \mathbb{L}_{\infty}^{k}([0,1])$ defines the set of functions $v:[0,1] \rightarrow K$ satisfying the following two conditions:

$$
\begin{aligned}
& \text { i) } \sum_{j=1}^{k} v^{j}(s)=\sum_{j=1}^{k} \mu^{j}(\tau) \text {, a.a. } s \in[0,1] \text {; } \\
& \text { ii) } \int_{0}^{1} v^{j}(s) d s=\mu^{j}(\tau), j=1, . ., k .
\end{aligned}
$$

Here, $\mu^{j}(\tau)=\mu^{j}(\{\tau\})$ is the value of $\mu^{j}$ at the single point set $\{\tau\}$, the symbol a.a.s means "almost all $s "$. Note that $W_{\tau}(\mu)=\{0\}$ whenever $\mu^{j}(\tau)=0, \forall j$.

The pair $\vartheta=\left(\mu ;\left\{v_{\tau}\right\}\right)$ is said to be impulsive control, provided $v_{\tau} \in W_{\tau}(\mu) \forall \tau \in T$. The family $\left\{v_{\tau}\right\}$ is called associated to the vector measure $\mu$. The variation $|\vartheta|$ of the impulsive control $\vartheta=$ $\left(\mu ;\left\{v_{\tau}\right\}\right)$ is the variation measure $|\mu|:=\sum_{j=1}^{k} \mu^{j}$.

Thus the impulsive control is constructed by the measure complemented with the associated family. The associated family is defined by the above relations i) and ii). As usual, the function $u(\cdot)$ in (5) defines conventional control and assumed to be measurable and essentially bounded with respect Lebesgue measure $\ell$. Let us now introduce the concept of trajectory.

Take an impulsive control $\vartheta=\left(\mu ;\left\{v_{\tau}\right\}\right)$, a number $\tau \in T$ and an arbitrary vector $x \in \mathbb{R}^{n}$. Denote by $\chi_{\tau}(\cdot)=\chi_{\tau}(\cdot, x)$ the solution to the following dynamical system

$$
\left\{\begin{array}{l}
\dot{\chi}_{\tau}(s)=g\left(\chi_{\tau}(s), \tau\right) v_{\tau}(s), s \in[0,1], \\
\chi_{\tau}(0)=x
\end{array}\right.
$$

The function of bounded variation $x(\cdot)$ on the interval $T$ is called solution to the differential equation in (5), corresponding to the control $(u, \vartheta)$ and the initial point $x_{0}$, if $x\left(t_{0}\right)=x_{0}$ and, for every $t \in\left(t_{0}, t_{1}\right]$,

$$
\begin{aligned}
& x(t)=x_{0}+\int_{t_{0}}^{t} f(x, u, \varsigma) d \varsigma+ \\
& +\int_{\left[t_{0}, t\right]} g(x, \varsigma) d \mu_{c}+\sum_{\tau \leq t}\left[x_{\tau}(1)-x\left(\tau^{-}\right)\right],
\end{aligned}
$$

where $x_{\tau}(\cdot):=\chi_{\tau}\left(\cdot, x\left(\tau^{-}\right)\right)$and the measure $\mu_{c}$ designates the continuous component of $\mu$. Note, that the sum in (7) is well defined since there is only a countable set of points $\tau$, where $v_{\tau}$ is nonzero.

The state constraints $\varphi(x, t) \leq 0$ in (5) should be understood in a wider sense than that of a conventional inequality. This is due to the presence of the impulsive component. Let us precise this meaning. Let us be given any admissible trajectory $x(\cdot)$ corresponding to the impulsive control $\vartheta$. Consider the variation measure $|\vartheta|$. Denote by $\operatorname{Ds}(\vartheta):=\{\tau \in T:|\vartheta|(\tau)>0\}$ the set of atoms of $|\vartheta|$ (impulses). Now, the inequality $\varphi(x, t) \leq 0$ has to be understood in the following generalized sense: $\varphi(x, t) \leq 0 \Leftrightarrow$

$$
\left\{\begin{aligned}
\varphi(x(t), t) \leq 0, & \text { a.a. } t \in T, \\
\varphi\left(x_{\tau}(s), \tau\right) \leq 0, & \text { a.a. } s \in[0,1], \forall \tau \in \operatorname{Ds}(\vartheta) .
\end{aligned}\right.
$$

The collection $(x, y, u, \vartheta)$ is called control process, if $d y=d|\vartheta|$ and (7) holds. A control process is said to be admissible, if all the constraints of problem (5) are satisfied. An admissible process $(\hat{x}, \hat{y}, \hat{u}, \hat{\vartheta})$ is said to be optimal if, for any admissible process $(x, y, u, \vartheta)$, the inequality $c(\hat{p}) \leq c(p)$ holds, where $\hat{p}=\left(\hat{x}\left(t_{0}\right), \hat{x}\left(t_{1}\right), \hat{y}\left(t_{1}\right)\right)$.

\section{Existence Theorem}

In the previous section we defined a certain extension of various control problems to the case of tra- 
jectories with discontinuities. Once the extension is defined, the main issue remains to understand whether and under what conditions, this extension is of interest in the sense that a solution exists to the extended problem. We shall now prove the existence under assumptions of Filippov's type.

Hereinafter, by modulus of a vector, we understand the sum of absolute values of the coordinates, i.e., $|x|=\sum_{i}\left|x^{i}\right|$. Then, $S_{\mathbb{R}^{k}}$, the unit sphere surface in $\mathbb{R}^{k}$, is

$$
S_{\mathbb{R}^{k}}:=\left\{x \in \mathbb{R}^{k}:|x|=1\right\} .
$$

\section{Theorem 1 Assume that:}

a) The sets $U$ and $S$ are compact.

b) The set $f(x, U, t)$ is convex for all $x, t$.

c) $\exists b>0: \varphi(x, t) \leq 0 \Rightarrow|x| \leq b$ for all $t$.

e) Problem (5) possesses at least one admissible process.

Then, there exists a solution to the problem (5).

Let us preface the proof with a simple exercise from convex analysis.

Lemma 2 Let the sets $A, B$ be convex. Then, the set

$$
C:=\bigcup_{\alpha \in[0,1]}(\alpha A+(1-\alpha) B)
$$

is also convex.

Proof: Let us fix a pair of points $\xi_{1}$, and $\xi_{2}$ in $C$ and $\beta \in(0,1)$. We need to show that $\beta \xi_{1}+(1-$ $\beta) \xi_{2} \in C$. By definition, we have:

$\xi_{1}=\alpha_{1} \xi_{1}^{A}+\left(1-\alpha_{1}\right) \xi_{1}^{B}, \quad \xi_{2}=\alpha_{2} \xi_{2}^{A}+\left(1-\alpha_{2}\right) \xi_{2}^{B}$, where $\xi_{i}^{A} \in A, \xi_{i}^{B} \in B$. Then,

$$
\begin{aligned}
\beta \xi_{1}+(1-\beta) \xi_{2}= & \beta \alpha_{1} \xi_{1}^{A}+\beta\left(1-\alpha_{1}\right) \xi_{1}^{B}+ \\
& (1-\beta) \alpha_{2} \xi_{2}^{A}+(1-\beta)\left(1-\alpha_{2}\right) \xi_{2}^{B} \\
= & {\left[\beta \alpha_{1} \xi_{1}^{A}+(1-\beta) \alpha_{2} \xi_{2}^{A}\right]+} \\
& {\left[\beta\left(1-\alpha_{1}\right) \xi_{1}^{B}+(1-\beta)\left(1-\alpha_{2}\right) \xi_{2}^{B}\right] . }
\end{aligned}
$$

If $\alpha_{1}=0$ and $\alpha_{2}=0$, or if $\alpha_{1}=1$ and $\alpha_{2}=1$, the desired inclusion is obvious due to convexity of the sets $A$, and $B$. Assume then that $\alpha_{1}$, and $\alpha_{2}$ are simultaneously either 0 or 1 . Let us put

$$
\begin{gathered}
\gamma_{A}=\beta \alpha_{1}+(1-\beta) \alpha_{2}, \text { and } \\
\gamma_{B}=\beta\left(1-\alpha_{1}\right)+(1-\beta)\left(1-\alpha_{2}\right) .
\end{gathered}
$$

Clearly, $\gamma_{A}$, and $\gamma_{B}$ are positive. From the above relation, we have

$$
\begin{aligned}
& \beta \xi_{1}+(1-\beta) \xi_{2}=\gamma_{A}\left[\frac{\beta \alpha_{1}}{\gamma_{A}} \xi_{1}^{A}+\frac{(1-\beta) \alpha_{2}}{\gamma_{A}} \xi_{2}^{A}\right]+ \\
& +\gamma_{B}\left[\frac{\beta\left(1-\alpha_{1}\right)}{\gamma_{B}} \xi_{1}^{B}+\frac{(1-\beta)\left(1-\alpha_{2}\right)}{\gamma_{B}} \xi_{2}^{B}\right] .
\end{aligned}
$$

Note that $\frac{\beta \alpha_{1}}{\gamma_{A}} \xi_{1}^{A}+\frac{(1-\beta) \alpha_{2}}{\gamma_{A}} \xi_{2}^{A} \in A$, and $\frac{\beta\left(1-\alpha_{1}\right)}{\gamma_{B}} \xi_{1}^{B}+\frac{(1-\beta)\left(1-\alpha_{2}\right)}{\gamma_{B}} \xi_{2}^{B} \in B$ due to convexity. It remains to ensure that $\gamma_{A}+\gamma_{B}=1$. Indeed,

$$
\begin{aligned}
\gamma_{A}+\gamma_{B}= & \beta \alpha_{1}+(1-\beta) \alpha_{2}+\beta\left(1-\alpha_{1}\right)+ \\
& +(1-\beta)\left(1-\alpha_{2}\right) \\
= & \beta \alpha_{1}+\alpha_{2}-\beta \alpha_{2}+\beta-\beta \alpha_{1}+1- \\
& -\beta-\alpha_{2}+\beta \alpha_{2} \\
= & 1 .
\end{aligned}
$$

Thus, $\beta \xi_{1}+(1-\beta) \xi_{2} \in C$.

Proof: The proof is based on the Lebesgue discontinuous change of time variable. Consider an auxiliary problem

$$
\left\{\begin{array}{l}
c(p) \rightarrow \min \\
\dot{x}=\alpha f(x, u, \chi)+(1-\alpha) g(x, \chi) v, \\
\dot{y}=1-\alpha \\
\dot{\chi}=\alpha, s \in\left[0, s_{1}\right] \\
p=\left(x_{0}, x_{1}, y_{1}\right) \in S \\
y(0)=0, \chi(0)=t_{0}, \chi\left(s_{1}\right)=t_{1} \\
\alpha \in[0,1], v \in K,|v|=1 \\
u \in U, \varphi(x, \chi) \leq 0
\end{array}\right.
$$

Unlike the problem (5) considered on the fixed time segment $\left[t_{0}, t_{1}\right]$ with the time variable $t$, the problem (8) is considered on the non-fixed time segment $\left[0, s_{1}\right]$ with the time variable $s$. However, problem (8) possesses control functions $u(s), v(s)$, $\alpha(s)$, where the scalar function $\alpha(s)$ is an auxiliary control, which are of conventional type, i.e., they are measurable and essentially bounded. Thus, problem (8) is conventional autonomous control problem with free time.

Let us show that the two problems, (5) and (8), are equivalent in the sense that for every admissible process $(x, y, u, \vartheta)$ of problem (5), there exists an admissible process $\left(\tilde{x}, \tilde{y}, \chi, \tilde{u}, v, \alpha, s_{1}\right)$ of problem (8), such that $c(p)=c(\tilde{p})$, where $p=$ $\left(x_{0}, x_{1}, y_{1}\right)$ and $\tilde{p}=\left(\tilde{x}_{0}, \tilde{x}_{1}, \tilde{y}_{1}\right)$, and vice-versa.

First, let us be given an admissible process $(x, y, u, \vartheta)$ of problem (5). Consider discontinuous time variable change

$$
\pi(t)=t-t_{0}+|\vartheta|\left(\left[t_{0}, t\right]\right) \text { as } t>t_{0}, \pi\left(t_{0}\right)=0 .
$$

Note that function $\pi(t)$ maps segment $T$ into $\left[0, s_{1}\right]$, where

$$
s_{1}=t_{1}-t_{0}+|\vartheta|(T) \text {. }
$$

Moreover, it maps $(\ell+|\vartheta|)$-measurable sets into $\ell$-measurable sets. It is easy to establish the existence of the inverse function $\theta(s):\left[0, s_{1}\right] \rightarrow T$ which has the following properties:

1) $\theta(s)$ is monotone increasing function on $\left[0, s_{1}\right]$ 
2) $\theta(s)$ is Lipschitz function since $|\theta(s)-\theta(t)| \leq$ $|s-t|, \forall s, t \in\left[0, s_{1}\right]$;

3) $\theta(s)=\tau, \forall s \in \Gamma_{\tau}, \forall \tau \in T$, where $\Gamma_{\tau}=$ $\left[\pi\left(\tau^{-}\right), \pi\left(\tau^{+}\right)\right]$.

By changing values of the function $u(t)$ on a zero $\ell$-measure set, we will ensure that $u(t)$ is measurable w.r.t. the measure $\ell+|\vartheta|$. Indeed, it is enough to put $u(t)=0, \forall t \in D$, where $D$ is an arbitrary set of points of zero measure that the singular component of the measure $|\hat{\vartheta}|$ maps onto a full measure set. On the other hand, since $\ell(D)=0$, the values of $u$ do not affect the evolution of the trajectory, and, hence, this change of values of the function $u(\cdot)$ is not restrictive.

Let us take $\tilde{u}(s)=u(\theta(s))$. The function $\tilde{u}$ is now $\ell$-measurable thanks to the properties of $u(t)$ and $\pi(t)$ stated above.

$$
\begin{gathered}
\alpha(s)= \begin{cases}m_{1}(\theta(s)), & \text { when } s \notin \bigcup_{\tau \in \operatorname{Ds}(\vartheta)} \Gamma_{\tau}, \\
0, & \text { otherwise, }\end{cases} \\
v(s)=\left\{\begin{array}{l}
\frac{m_{2}(\theta(s))}{1-\alpha(s)}, \text { if } s \notin \bigcup_{\tau \in \operatorname{Ds}(\vartheta)} \Gamma_{\tau} \text { and } \alpha(s)<1, \\
\ell^{-1}\left(\Gamma_{\tau}\right) v_{\tau}\left(\xi_{\tau}(s)\right), \text { when } s \in \Gamma_{\tau},
\end{array}\right.
\end{gathered}
$$

where, $m_{1}$ and $m_{2}$ are the Radon-Nykodim derivatives of the measures $\ell$ and $\mu_{c}$ with respect to $\ell+|\vartheta|, \xi_{\tau}(s):=\frac{s-\pi\left(\tau^{-}\right)}{\ell\left(\Gamma_{\tau}\right)}: \Gamma_{\tau} \rightarrow[0,1]$. When $\alpha(s)=1$, the values of $v(s)$ may be taken as arbitrary unit vectors from $K$, since the trajectory $x(\cdot)$ does not depend on those values. Note that $v(s) \in K$ and $|v(s)|=1$ due to $m_{1}(t)+\left|m_{2}(t)\right|=1$ a.a. $t \notin \operatorname{Ds}(\vartheta)$ w.r.t. the measure $\ell+|\vartheta|$.

By definition, it follows $\theta(s)=t_{0}+\int_{0}^{s} \alpha(\varsigma) d \varsigma$. Therefore, $\chi(s)=\theta(s)$. By performing the variable change in (7), it is easy to see that the trajectory $\tilde{x}(\cdot)$, solution to the dynamical system (8) corresponding to the just constructed collection $\left(x_{0}, \tilde{u}, v, \alpha, s_{1}\right)$, exists and it is exactly the function

$x^{\operatorname{ext}}(s):= \begin{cases}x_{\tau}\left(\xi_{\tau}(s)\right) & \text { if } \exists \tau \in \operatorname{Ds}(\vartheta): s \in \Gamma_{\tau}, \\ x(\theta(s)) & \text { otherwise. }\end{cases}$

Then, $\tilde{x}\left(s_{1}\right)=x\left(t_{1}\right)$. By construction, we also have that $\tilde{y}\left(s_{1}\right)=|\vartheta|(T)=y\left(t_{1}\right)$. Therefore, $c(p)=c(\tilde{p})$ and $\tilde{p} \in S$. Besides $\varphi(\tilde{x}(s), \chi(s))=$ $\varphi\left(x^{\operatorname{ext}}(s), \theta(s)\right) \leq 0$ in view of definition of the state constraints. So, the process constructed above is admissible to problem (8) and the cost function takes the same value, $c(p)$.

Conversely, let us be given an admissible process $\left(\tilde{x}, \tilde{y}, \chi, \tilde{u}, v, \alpha, s_{1}\right)$ of problem (8). The function $\chi(s)$ is the inverse of some discontinuous time variable change $\tilde{\pi}: T \rightarrow\left[0, s_{1}\right]$. The function $\tilde{\pi}$ is uniquely defined as the one that satisfies $\tilde{\pi}(\chi(s))=s$, a.a. $s$ such that $\alpha(s)>0, \tilde{\pi}\left(t_{0}\right)=0$, $\tilde{\pi}\left(t_{1}\right)=s_{1}$, and $\tilde{\pi}(t)$ is right-continuous in $\left(t_{0}, t_{1}\right)$. Once $\tilde{\pi}$ is determined, we find the measure $\mu$ via its distribution function:

$$
F(t, \mu)=\int_{0}^{\tilde{\pi}(t)}(1-\alpha(s)) v(s) d s
$$

Let us take $u(t)=\tilde{u}(\tilde{\pi}(t)), v_{\tau}(s)=\ell\left(\tilde{\Gamma}_{\tau}\right) v\left(\tilde{\gamma}_{\tau}(s)\right)$, where

$$
\begin{gathered}
\tilde{\Gamma}_{\tau}=\left[\tilde{\pi}\left(\tau^{-}\right), \tilde{\pi}\left(\tau^{+}\right)\right], \\
\tilde{\gamma}_{\tau}(s)=\ell\left(\tilde{\Gamma}_{\tau}\right) s+\tilde{\pi}\left(\tau^{-}\right):[0,1] \rightarrow \tilde{\Gamma}_{\tau} .
\end{gathered}
$$

Let $x(\cdot)$ be the solution defined by (7). It follows right from the change of variable that $x\left(t_{1}\right)=$ $\tilde{x}\left(s_{1}\right), y\left(t_{1}\right)=\tilde{y}\left(s_{1}\right)$. Clearly, the endpoint and the state constraints are satisfied. So, the constructed process is admissible to problem (5), whereas the cost function takes on the same value $c(\tilde{p})$.

Thus, we have shown that the two problems (5) and (8), are equivalent. Therefore, if a solution exists to one of the problem, then it also exists to the other.

Let us check the existence of solution to the auxiliary problem (8). Observe that the velocity set of (8), that is,

$$
\begin{gathered}
\bigcup_{\alpha \in[0,1]} \alpha(f(x, U, t), 0,1)+ \\
+(1-\alpha)\left(g(x, t)\left(K \cap S_{\mathbb{R}^{k}}\right), 1,0\right) \subseteq \mathbb{R}^{n+2},
\end{gathered}
$$

is convex due to the assumption b) of the theorem, the convexity of the set $K \cap S_{\mathbb{R}^{k}}$, and also to Lemma 2. This, coupled with the other assumptions a), c), e) allow us to apply the classic Filippov's existence theorem to the problem (8) which is valid under the considered conditions. So, the solution to the auxiliary problem exists. Then, it also exists to the original problem (5).

Above, we implicitly used the fact that the endpoint constraints in (8) and the assumption a) imply $s_{1} \leq$ const. Indeed, for the trajectory $y$ in (8), we have:

$$
\begin{gathered}
y\left(s_{1}\right)=\int_{0}^{s_{1}}(1-\alpha) d s=s_{1}-\left(t_{1}-t_{0}\right) \stackrel{\text { a) }}{\Longrightarrow} \\
s_{1} \leq \text { const } .
\end{gathered}
$$

The assumption d) was used implicitly while performing the discontinuous variable change.

The proof is complete.

\section{ACKNOWLEDGMENTS}

The first and fourth authors were supported by CNPq (Brazil) "Sem Fronteiras" grant no 401689/2012-3. The third author was supported by FCT (Portugal) grants Incentivo/EEI/UI0147/2013, and PEstOE/EEI/UI0147/2011 with its extension to 2013. 